\title{
Determining the fraction of dust heating from young and old stellar populations with $3 \mathrm{D}$ dust radiative transfer
}

\author{
Giovanni Natale ${ }^{1,2}$, Cristina C. Popescu ${ }^{1,2}$, Richard. J. Tuffs ${ }^{2}$, Victor \\ P. Debattista ${ }^{1}$, Jörg Fischera ${ }^{2}$ and Meiert W. Grootes ${ }^{2}$ \\ ${ }^{1}$ Jeremiah Horrocks Institute, University of Central Lancashire, Preston, PR1 2HE, UK \\ ${ }^{2}$ Max Planck Institute für Kernphysik, Saupfercheckweg 1, D-69117 Heidelberg, Germany
}

\begin{abstract}
A major difficulty hampering the accuracy of UV/optical star formation rate tracers is the effect of interstellar dust, absorbing and scattering light produced by both young and old stellar populations (SPs). Although empirically calibrated corrections or energy balance SED fitting are often used for fast de-reddening of galaxy stellar emission, eventually only radiative transfer calculations can provide self-consistent predictions of galaxy model spectra, taking into account important factors such as galaxy inclination, different morphological components, nonlocal heating of the dust and scattered radiation. In addition, dust radiative transfer can be used to determine the fraction of monochromatic dust emission powered by either young or old SPs. This calculation needs to take into account the different response of the dust grains to the UV and optical radiation field, depending on the grain size and composition. We determined the dust heating fractions, on both global and local scales, for a high-resolution galaxy model by using our 3D ray-tracing dust radiative transfer code "DART-Ray". We show the results obtained using this method and discuss the consequences for star formation rate indicators.
\end{abstract}

Keywords. radiative transfer, n-body simulations, dust, extinction, galaxies

\section{Introduction}

The propagation of stellar radiation in a galaxy is a highly non-local process. In fact, the appearance of a galaxy at UV and optical wavelengths, a regime dominated by stellar emission, is strongly dependent on the absorption of stellar radiation as well as scattering in the direction of the observer. On the other hand, in the infrared (IR), the dust emission depends on the amount of dust heating which can be in principle provided by all stars in a galaxy, including those located at large distances from the dust. For these reasons, dust radiative transfer modelling of galaxies is essential to correctly interpret their observed spectral energy distributions. For a given galaxy model, dust radiative transfer calculations can also be used to determine, at each position, the dust emission powered by radiation produced by young and old stellar populations (SP). Once an age criteria has been defined to identify "young" and "old" stars, this can be done by following the procedure described in section 3 of Natale et al. (2015), which is an extension of the method of Popescu et al. 2000 (see also Popescu et al. 2011). We applied this technique to the galaxy simulation described in Cole et al. (2014) by using our 3D ray-tracing radiative transfer code DART-Ray (Natale et al. 2014).

\section{Results}

The panel on the left of Fig. 1 shows, for the simulated galaxy, the relative contribution to the spatially integrated dust emission powered by young SPs (defined as stars 


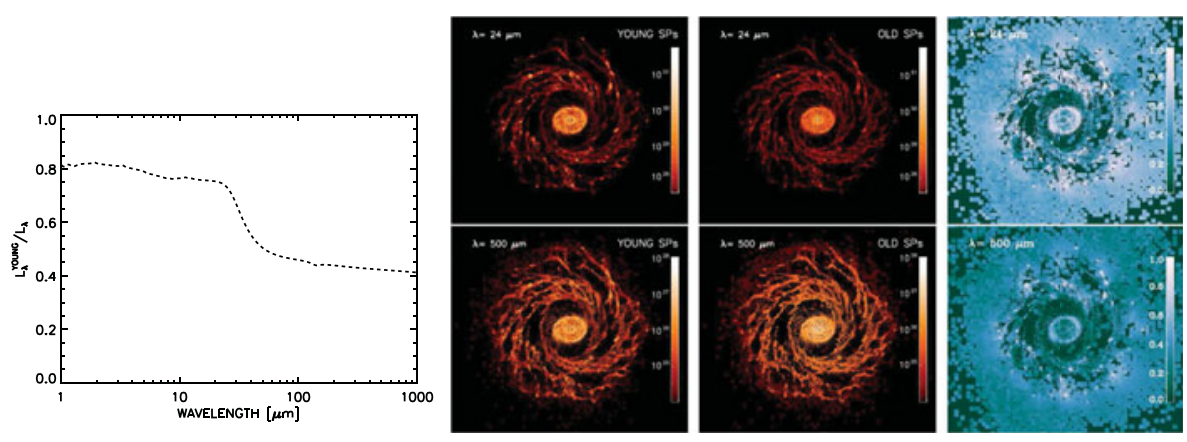

Figure 1. Left: Relative contribution to the spatially integrated dust emission powered by young SP at each IR wavelength; Right, top row: $24 \mu \mathrm{m}$ dust emission powered by young and old SP for the face-on view of the simulated galaxy (units are erg/( $\left.\mathrm{s} \AA \mathrm{sr} \mathrm{pc}^{2}\right)$ ); relative fraction of 24 $\mu m$ dust emission powered by young SPs. Bottom row: same but for $\lambda=500 \mu m$.

with ages $<1.5 \times 10^{8} \mathrm{yr}$ ) at each IR wavelength. One can notice that at the shortest wavelengths up to about $30 \mu \mathrm{m}$, the heating of the dust emission is dominated by young SPs at the level of about $80 \%$. At longer wavelengths, the young SP relative contribution first decreases rapidly between $30-60 \mu m$ and then it stabilizes at around $40-50 \%$ in the far-IR. The panels in the top right-hand side of Fig. 1 show the face-on $24 \mu \mathrm{m}$ dust emission contributed by young and old SPs as well as the relative contribution due to the young SPs. One can see that the morphology of the dust emission powered by young SPs is more clumpy than that powered by old SPs. The clumpy emission is associated with regions of recent star formation. At $24 \mu \mathrm{m}$ both the diffuse and clumpy emission are dominated by heating from young SPs. Instead at $500 \mu \mathrm{m}$ (see bottom right-hand side panels in Fig.1), the diffuse dust emission becomes slightly dominated by old SP heating, while the clumpy emission remains dominated by young SP heating.

Star formation rate tracers based on the measurement of the dust emission from galaxies are more reliable when the contamination due to old SP heating is reduced. Our results suggest that for a galaxy as a whole, the MIR dust emission is more prone to star formation rate measurement than the FIR emission, since for the latter the old SP contamination is much higher. For star formation rate measurements within galaxies, we found that only close to recent star formation sites the dust emission is clearly dominated by young SPs. Instead the relative contribution of the young SPs in heating the diffuse dust emission can vary substantially both in position within a galaxy and at different wavelengths.

G.N. and C.C.P. would like to acknowledge support from the Leverhulme Trust research project grant RPG-2013-418. V.P.D. is supported by STFC Consolidated grant \# ST/M000877/1

\section{References}

Cole, D., Debattista, V. P., Erwin, P., Earp, S. W. F., \& Roskar, R., 2014, MNRAS, 445, 3352

Natale, G., Popescu, C. C., Tuffs, R. J., \& Semionov, D. 2014, MNRAS, 438, 3137

Natale, G. , Popescu, C. C., Tuffs, R.. J., et al. 2015, MNRAS, 449, 243

Popescu, C. C., Misiriotis, A., Kylafis, N. D., Tuffs, R. J., \& Fischera, J., 2000, A\&SA, 362, 138

Popescu, C. C., Tuffs, R. J., Dopita, M. A., Fischera, J., et al. 2011, A\&̈A, 527, 109 